\title{
Efeito da acidificação nas propriedades físico-químicas e funcionais do amido de sementes de manga (Mangifera indica L.), variedade Tommy Atkins ${ }^{1}$
}

\author{
Marianne Louise Marinho Mendes*2, Ana Paula Loura Ribeiro ${ }^{3}$, Elisandra Costa Almeida \\ http://dx.doi.org/10.1590/0034-737X201562030001
}

\section{RESUMO}

No processamento industrial da manga, os subprodutos, caroço e casca são, normalmente, descartados sem o devido aproveitamento. O objetivo deste trabalho foi investigar as características físico-químicas e funcionais dos amidos nativo e acidificado do caroço (amêndoa) de manga, da variedade Tommy Atkins, pesquisando uma nova fonte de amido e formas de melhorá-la e contribuir para a utilização de resíduos da indústria alimentícia. As amêndoas foram retiradas manualmente dos caroços de manga e procedeu-se a extração do amido. $\mathrm{O}$ amido nativo foi acidificado, sendo o agente promotor da modificação o ácido clorídrico. Verificou-se a composição química do amido nativo. Determinaram-se também as propriedades funcionais e de pasta do amido nativo e do acidificado. Para a composição centesimal, o amido nativo apresentou $71,56 \%$ de amido, 7,30\% de lipídeos e 5,6\% de proteínas. Os amidos nativo e acidificado demonstraram baixa resistência à elevação da temperatura, com intumescimento máximo a $75^{\circ} \mathrm{C}$, sendo seus valores 9,395 e $6,861 \mathrm{~g} / \mathrm{g}$, respectivamente. A solubilidade mostrou-se crescente, à medida que houve elevação da temperatura, sendo seu valor máximo $48,03 \%$ para o amido acidificado. $\mathrm{O}$ amido nativo expôs maior capacidade de absorção de água, óleo e claridade da pasta. O referido amido ainda apresentou maior pico de viscosidade, viscosidade mínima, quebra de viscosidade, viscosidade final, retrogradação e temperatura de pasta do que os do amido acidificado. $\mathrm{O}$ amido nativo pode ser indicado para compor sopas desidratadas e produtos cárneos, enquanto o acidificado pode ser usado em alimentos congelados e refrigerados e na indústria de balas.

Palavras chave: amido modificado, viscosidade, RVA.

\section{ABSTRACT \\ Effect of acidification on the physicochemical and functional properties of mango seed starch (Mangifera indica L.), Tommy Atkins Variety}

The industrial processing of mango generates by-products such as kernel and peel that are usually discarded without proper utilization. The objective of this study was to investigate the physicochemical and functional properties of native and acidified starch separated from mango kernels of variety Tommy Atkins, searching for a new source of starch, ways to improve it and contribute to the utilization of residues from the food industry. Kernels were removed manually and starch was extracted. The native starch was acidified with hydrochloric acid and its chemical composition was -determined. Functional and paste properties of native and acidified starch was also determined. The chemical

\footnotetext{
Submetido em 04/11/2013 e aprovado em 26/02/2015.

' Este trabalho é parte da tese de doutorado da primeira autora.

${ }^{2}$ Universidade de Pernambuco, Petrolina, Pernambuco, Brasil. marianne.marinho@upe.br.

${ }^{3}$ Universidade Federal da Paraíba, Departamento de Tecnologia Química e de Alimentos, João Pessoa, Paraíba, Brasil. anapaulaloura@ @mail.com.

${ }^{4}$ Universidade Federal da Paraíba, Departamento de Tecnologia Química e de Alimentos, João Pessoa, Paraíba, Brasil. elisandra.quimica@gmail.com

*Autora para correspondência: marianne.marinho@upe.br
} 
composition of the native starch consisted of $71.56 \%$ starch, $7.30 \%$ lipids and $5.6 \%$ protein. Native and acidified starches showed low resistance to high temperature, with maximum swelling at $75^{\circ} \mathrm{C}$ of 9.395 and $6.861 \mathrm{~g} / \mathrm{g}$, respectively. The solubility increased with temperature achieving the maximum of $48.03 \%$ for acidified starch. The native starch had greater capacity to absorb water and oil and greater paste clarity. It also showed the highest peak viscosity, hold on, breakdown, final viscosity, setback and paste temperature than the acidified starch. The native starch can be indicated to make dehydrated soups and meat products, while the acidified starch can be used in frozen and chilled food and candy industry.

Key words: modified starch, viscosity, RVA.

\section{INTRODUÇÃO}

A manga (Mangifera indicans L.) pertence à família Anacardiaceae e figura entre as frutas tropicais de maior expressão econômica nos mercados brasileiros e internacionais (Brandão et al., 2003). Dependendo da variedade, as amêndoas de seus caroços contêm cerca de $6,0 \%$ de proteínas, $11 \%$ de lipídeos, $77 \%$ de carboidratos, $2 \%$ de fibras e $2 \%$ de cinzas, com base em seu peso seco (Zein El-Bagoury \& Kassab, 2005). Atualmente, a manga variedade Tommy Atkins é a mais produzida e a que apresenta maior participação no volume comercializado no mundo, principalmente por sua coloração intensa, produções elevadas e resistência ao transporte a longas distâncias (Costa \& Santos, 2004).

De acordo com Beemiller \& Wistler (2009), o amido é constituinte da maioria das plantas superiores, sendo acumulado transitoriamente nos cloroplastos, durante o dia, quando a fotossíntese excede a demanda de assimilação pela planta, e transladado, durante a noite, para outras partes da planta, na forma de açúcares. Órgãos de reserva da planta, como sementes, raízes e frutos, mantêm o amido armazenado para ser consumido na germinação e desenvolvimento de uma nova planta. Nas indústrias agroalimentares, os amidos e derivados são utilizados como ingredientes, componentes básicos ou aditivos, adicionados em baixas quantidades, para melhorar a fabricação, apresentação ou conservação (Serrano \& Franco, 2005) .

Entretanto, muitos amidos, em sua forma nativa, apresentam limitações que os tornam menos adequados para a diversidade de aplicações requeridas. Por esta razão, algumas vezes o amido, utilizado como alimento ou para outros fins industriais, tem que ser modificado (química, física, enzimaticamente) antes de sua utilização, com o objetivo de melhorar e aperfeiçoar as propriedades físicas do polímero, em consonância com a sua aplicação específica (Bertolini, 2010).
Pode-se dizer, de forma geral, que amidos modificados por ácidos são aqueles que foram suspensos em uma solução ácida, em temperatura abaixo de seu ponto de gelatinização (Wang \& Wang, 2003). Esta modificação pode ser controlada pela concentração do ácido, tempo de reação e temperatura (Wang et al., 2003).

$\mathrm{O}$ amido modificado por ácido, como não apresenta mudanças significativas na sua forma granular, tem birrefringência similar e a mesma insolubilidade em água fria que as do amido nativo. No entanto, apresenta menor viscosidade de pasta quente, maior relação viscosidade fria/viscosidade a quente (poder de geleificação), ou seja, menor inchamento dos grânulos durante a gelatinização em água quente, menor viscosidade intrínseca, alteração da solubilidade em água a temperaturas inferiores à de gelatinização e maior temperatura de gelatinização (Zambrano \& Camargo, 2001) .

Realizou-se, neste trabalho, a investigação da composição centesimal do amido nativo extraído da amêndoa do caroço (semente) de manga, variedade Tommy Atkins. Observaram-se, também, as propriedades funcionais e de pasta desse amido nativo e de seu derivado acidificado com o intuito de se estudar uma nova fonte em potencial de amido e maneiras de aperfeiçoá-la, bem como para contribuir ecologicamente para o aproveitamento de resíduos da indústria alimentícia.

\section{MATERIAL E MÉTODOS}

A matéria-prima alvo desta pesquisa foi o amido extraído de amêndoas, retirado dos caroços de manga (Mangifera indicans L.), da variedade "Tommy Atkins". As mangas foram adquiridas na Empresa Paraibana de Abastecimento e Serviços Agrícolas (EMPASA), na cidade de João Pessoa, PB.

\section{Extração do amido}

As amêndoas dos caroços de manga foram retiradas manualmente e a seguir procedeu-se à extração do ami- 
do, segundo o método descrito por Loos et al. (1981). $\mathrm{O}$ amido obtido foi acondicionado em um recipiente hermeticamente fechado, sob refrigeração, até o momento de realização das análises, que ocorreram no período de seis meses.

\section{Composição centesimal}

O extrato amiláceo nativo (bruto) foi avaliado quanto à umidade, cinzas, lipídeos, proteínas e teor de amido. Os métodos foram descritos pelo Instituto Adolfo Lutz (2008).

\section{Tratamento ácido}

O tratamento ácido empregado nesta pesquisa foi o mesmo usado por Lawal \& Adebowale (2005). Diluíram-se $100 \mathrm{~g}$ de amido nativo em $500 \mathrm{ml}$ de ácido clorídrico a $0,15 \mathrm{M}$, agitou-se a solução por oito horas, mantendo-se a temperatura em $50{ }^{\circ} \mathrm{C}$. Em seguida, filtrouse o amido modificado, sendo este lavado quatro vezes com água destilada, e secado em estufa com circulação de ar, a $45^{\circ} \mathrm{C}$, por 24 horas. Após isso, o amido obtido foi tamisado (200 mesh) e armazenado em recipiente fechado, sob refrigeração.

\section{Poder de intumescimento e solubilidade}

Foram determinados o poder de intumescimento e a solubilidade em função da temperatura e do $\mathrm{pH}$, dos amidos nativo e acidificado. Em função da temperatura, essas variáveis foram determinadas conforme metodologia descrita por Leach et al. (1959).

\section{Capacidade de absorção de água e óleo}

Para esta determinação, foi usado o método de Beuchat (1977), adicionando-se $10 \mathrm{ml}$ de água (destilada) ou do óleo (óleo de soja Soya, Bunge - Indústria Brasileira) a $1 \mathrm{~g}$ da amostra, sendo esta análise realizada nos amidos nativo e acidificado.

\section{Transparência da pasta}

A transparência da pasta foi determinada, para ambos os amidos, por transmitância (\%T) a $650 \mathrm{~nm}$, com o método descrito por Craig et al. (1989).

\section{Características das pastas de amido}

A determinação das propriedades de pasta ocorreu, para os amidos nativo e acidificado, em equipamento RVA - Rapid Visco Analyser (Newport Scientific).

As amostras, na concentração de $10 \%$, foram submetidas à temperatura de $50{ }^{\circ} \mathrm{C}$, por 1 minuto e, depois, aquecida até $95^{\circ} \mathrm{C}$, em uma taxa de $6{ }^{\circ} \mathrm{C} / \mathrm{min}$, permanecendo nessa temperatura por 5 minutos. Em seguida, resfriaram-se as amostras até $50{ }^{\circ} \mathrm{C}$, também em uma taxa de $6{ }^{\circ} \mathrm{C} / \mathrm{min}$. Nessa análise, foram obtidos os valores de temperatura de pasta, viscosidade máxima, mínima e final, quebra de viscosidade (diferença entre a viscosidade máxima e a mínima) e tendência à retrogradação (diferença entre a viscosidade final e mínima).

\section{Análise estatística}

Aos dados da composição centesimal e das propriedades funcionais, foi aplicada a estatística descritiva com observação das médias e dos desvios-padrão de três repetições. Às propriedades funcionais aplicou-se a análise de variância (ANOVA) e o teste de Tukey, a 5\% de significância, para comparação entre as médias obtidas. As análises foram realizadas pelo programa estatístico SigmaStat 3.5.

\section{RESULTADOS}

\section{Composição centesimal do amido}

$\mathrm{Na}$ Tabela 1, apresentam-se os resultados referentes à composição centesimal do amido extraído do caroço (semente) de manga, var. Tommy Atkins

\section{Propriedades funcionais}

Na Tabela 2, observam-se os resultados referentes ao poder de intumescimento (PI) e à solubilidade (S), conforme a temperatura. Após a acidificação, as propriedades do amido mostraram-se alteradas. Pode-se verificar que, após a temperatura de $75^{\circ} \mathrm{C}$, os amidos mostraram diminuição do PI, porém os valores de $\mathrm{S}$ elevaram-se à medida que aumentou a temperatura. $\mathrm{O}$ maior PI foi detectado no amido nativo, a $75{ }^{\circ} \mathrm{C}(9,395 \pm 0,396$ $\mathrm{g} / \mathrm{g}) \mathrm{e}$, a maior $\mathrm{S}$, no amido acidificado, a $95{ }^{\circ} \mathrm{C}(48,03 \pm$ $0,075 \%)$.

Os valores para capacidade de absorção de água (CAA) e óleo (CAO) estão expostos na Figura 1. Por meio deles, pode-se identificar que o amido nativo mostrou maior valor, tanto para a CAA como para a CAO.

Quanto à transparência da pasta, seus valores podem ser observados na Figura 2. O amido acidificado apresentou menor valor de claridade da pasta do que o amido nativo.

Tabela 1: Composição centesimal do amido nativo extraído das amêndoas das sementes de manga

\begin{tabular}{lc}
\hline Constituintes & Quantidades $(\mathbf{g} / \mathbf{1 0 0 g})$ \\
\hline Amido & $71,56 \pm 1,03$ \\
Umidade & $10,30 \pm 0,01$ \\
Lipídeos & $7,30 \pm 0,20$ \\
Proteínas $(\mathrm{N}$ x 6,25) & $5,60 \pm 0,25$ \\
Cinzas & $0,40 \pm 0,17$ \\
Outros componentes (por diferença) & 4,84 \\
\hline
\end{tabular}

* Cada valor representa a média e o desvio-padrão de três determinações. 


\section{Características das pastas de amido}

Na Tabela 3, podem-se verificar os valores de propriedades de pasta avaliados para os dois amidos deste estudo. Observa-se que o amido nativo apresentou maiores valores para todos os parâmetros de propriedades de pasta avaliados (letras diferentes entre colunas), exceto para a temperatura de pasta, que não apresentou diferença significativa entre as duas amostras (letras iguais entre colunas). Por isto, o amido nativo apresentou maior pico de viscosidade, porém o amido acidificado mostrou uma menor retrogradação.

\section{DISCUSSÃO}

$\mathrm{O}$ valor de amido encontrado foi inferior ao encontrado por Rengsutthi \& Charoenrein (2011) $(99,65 \mathrm{~g} /$ $100 \mathrm{~g}$ ), estudando amido de sementes de jaca. Batista $e t$ al. (2010) encontraram valores de 86,97 a $86,62 \mathrm{~g} / 100$ g para carboidratos em amidos convencionais de milho e trigo, respectivamente. Os primeiros autores encontraram ainda, para amido da semente de jaca, valores de proteínas $(0,09 \mathrm{~g} / 100 \mathrm{~g})$ e lipídeos $(0,03 \mathrm{~g} / 100 \mathrm{~g})$ bem inferiores aos detectados no amido deste trabalho, porém obtiveram o mesmo valor de cinzas $(0,04 \mathrm{~g} / 100 \mathrm{~g})$ deste estudo.
De acordo com Bertolini (2010), o amido bruto é constituído de carboidratos, bem como por substâncias como lipídeos, proteínas e cinzas. A quantidade destes constituintes dependerá da composição da planta e dos métodos de extração e de purificação. Tem forte influência também a parte da planta da qual o amido foi extraído.

O poder de intumescimento e a solubilidade são determinados em temperaturas elevadas, promovendo a quebra de pontes de hidrogênio, ocorrendo o intumescimento dos grânulos e o aumento da solubilidade do amido. Ambos contribuem para importantes características da maioria dos produtos ricos em amido, como as propriedades de pasta e o comportamento reológico durante o aquecimento em excesso de água (Tongdang, 2008).

Com relação à temperatura, os amidos nativo e acidificado analisados alcançaram valores de intumescimento máximo a $75{ }^{\circ} \mathrm{C}$. Após isto, seus valores de PI iniciaram um declínio. Isto pode demonstrar que os grânulos desses amidos têm uma baixa resistência à elevação da temperatura, ocorrendo, muito provavelmente, seu rompimento com a elevação da mesma, contribuindo este fato também para o aumento da solubilidade. Hashim et al. (1992) justificam este fato dizendo que, na faixa de

Tabela 2: Poder de intumescimento (PI) e solubilidade (S), conforme a temperatura e o pH, do amido nativo e acidificado da amêndoa do caroço de manga

\begin{tabular}{|c|c|c|c|c|}
\hline \multirow{2}{*}{$\mathbf{T}\left({ }^{\circ} \mathrm{C}\right)$} & \multicolumn{2}{|c|}{ PI (g/g) } & \multicolumn{2}{|c|}{$\mathrm{S}(\%)$} \\
\hline & Amido nativo & Amido acidificado & Amido nativo & Amido acidificado \\
\hline 55 & $1,286 \pm 0,080^{\mathrm{Aa}}$ & $1,321 \pm 0,048^{\mathrm{Aa}}$ & $1,43 \pm 0,006^{\text {Aa }}$ & $1,37 \pm 0,011^{\mathrm{Aa}}$ \\
\hline 65 & $1,480 \pm 0,034^{\mathrm{Ab}}$ & $1,670 \pm 0,044^{\mathrm{Ab}}$ & $2,40 \pm 0,020^{\mathrm{Ab}}$ & $1,97 \pm 0,028^{\mathrm{Ab}}$ \\
\hline 75 & $9,395 \pm 0,396^{\mathrm{Ac}}$ & $6,861 \pm 0,503^{\mathrm{Bc}}$ & $3,87 \pm 0,012^{\mathrm{Ac}}$ & $8,13 \pm 0,030^{\mathrm{Bc}}$ \\
\hline 85 & $7,853 \pm 0,541^{\mathrm{Ad}}$ & $4,085 \pm 0,243^{\mathrm{Bd}}$ & $5,47 \pm 0,057^{\mathrm{Ad}}$ & $38,57 \pm 0,092^{\mathrm{Bd}}$ \\
\hline 95 & $4,955 \pm 0,494^{\mathrm{Ae}}$ & $0,899 \pm 0,011^{\mathrm{Be}}$ & $33,90 \pm 0,555^{\mathrm{Ae}}$ & $48,03 \pm 0,075^{\mathrm{Be}}$ \\
\hline
\end{tabular}

* Cada valor representa a média e o desvio-padrão de três determinações.

Diferença significativa entre colunas para uma mesma propriedade - letras maiúsculas diferentes.

Diferença significativa entre linhas para uma mesma propriedade - letras minúsculas diferentes.

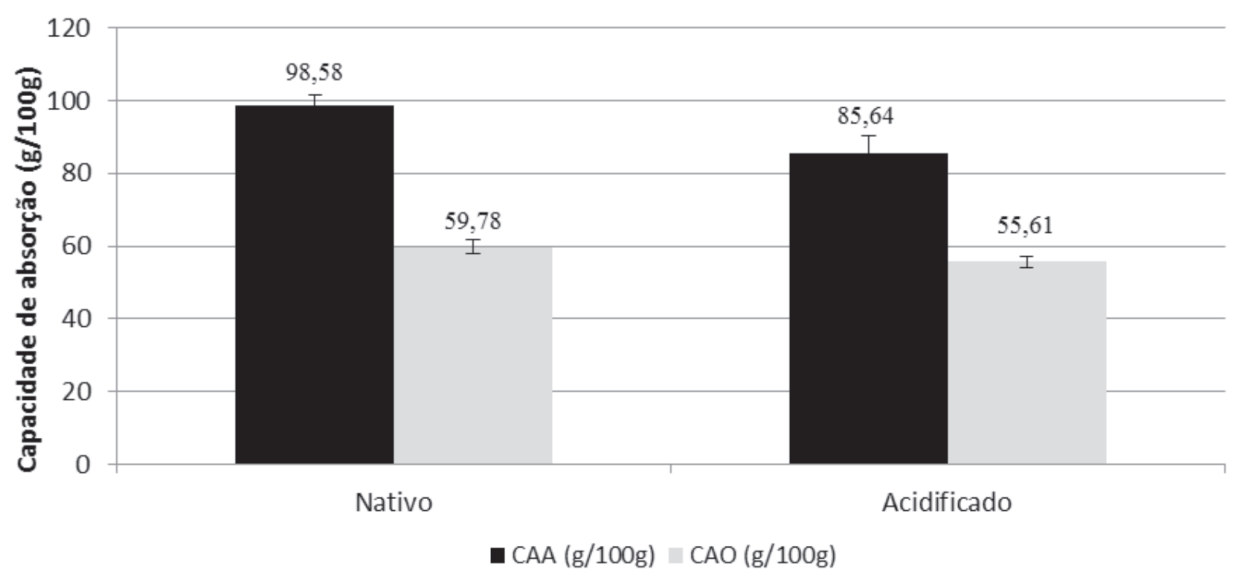

Figura 1: Capacidade de absorção de água e óleo dos amidos nativo e acidificado das amêndoas dos caroços de manga.

Rev. Ceres, Viçosa, v. 62, n.3, p. 225-232, mai-jun, 2015 
temperatura de gelatinização, o grânulo de amido tem um limitado intumescimento, em que apenas uma pequena quantidade desse polímero é solubilizada, mas, em temperaturas superiores, uma grande quantidade de amido é lixiviada dos grânulos.

Verifica-se que o amido nativo mostrou valor de PI menor do que a maioria dos amidos nativos de outras origens, relatados na literatura. Kaur et al. (2004), estudando amido do caroço de cinco variedades de manga indianas, observaram valores de PI entre 18,0 e 19,7 g, superiores, portanto, aos desta pesquisa. Os mesmos autores destacam, ainda, que o baixo PI pode ser atribuído à presença de lipídeos no amido, que formam complexo com a amilose. Osundahunsi \& Mueller (2011), pesquisando propriedades de amido de duas variedades de mandioca, encontraram amidos nativos com PI de 21,3 e 29,6 g/g. No entanto, Adebowale et al. (2005) encontraram, para o amido de fruta-pão, PI de $6,40 \mathrm{~g} / \mathrm{g}$ a $80^{\circ} \mathrm{C}$. Tem sido reportado que o PI depende da capacidade de retenção de água das moléculas de amido por pontes de hidrogênio. As pontes de hidrogênio estabilizam as duplas hélices das estruturas cristalinas dos grânulos de amido. Estas são quebradas durante a gelatinização e passam a ter ligações de hidrogênio com a água, sendo o PI regulado pela cristalinidade do amido. Além disso, o grau de ramificação da cadeia amilácea também pode influ- enciar o grau de inchamento e a solubilidade deste amido (Anggraini et al., 2009).

$\mathrm{O}$ amido acidificado do caroço de manga de forma geral mostrou valores de PI menores do que o nativo. Singh et al. (2011), estudando tratamentos ácidos em amido de sorgo, observaram que, à medida que se aumentava a molaridade da solução de ácido clorídrico, usada na acidificação, ocorria diminuição dessa propriedade, sendo também verificado que o amido de sorgo acidificado tinha sempre menor PI que o nativo, independentemente da concentração da solução ácida usada. Os referidos autores encontraram valor de PI variando entre 8,6 g/g ( $\mathrm{HCl} 1 \mathrm{M})$ e $10,6 \mathrm{~g} / \mathrm{g}(\mathrm{HCl} 0,1 \mathrm{M})$.

Com relação à solubilidade, à medida que ocorreu aumento da temperatura, a solubilidade dos amidos avaliados também aumentou, sendo que o amido acidificado apresentou maior solubilidade do que o nativo. Kaur et al. (2004), estudando amido do caroço de cinco variedades de mangas indianas, encontraram valores de solubilidade, a $95{ }^{\circ} \mathrm{C}$, menores do que o encontrado para o amido nativo desta pesquisa. Bello-Pérez et al. (2006), analisando amido de pinhão, observaram que sua solubilidade aumentava conforme a temperatura, com variação de 5 a 20\%. Já Osundahunsi \& Mueller (2011) obtiveram 3,34\% de solubilidade para o amido nativo de mandioca.

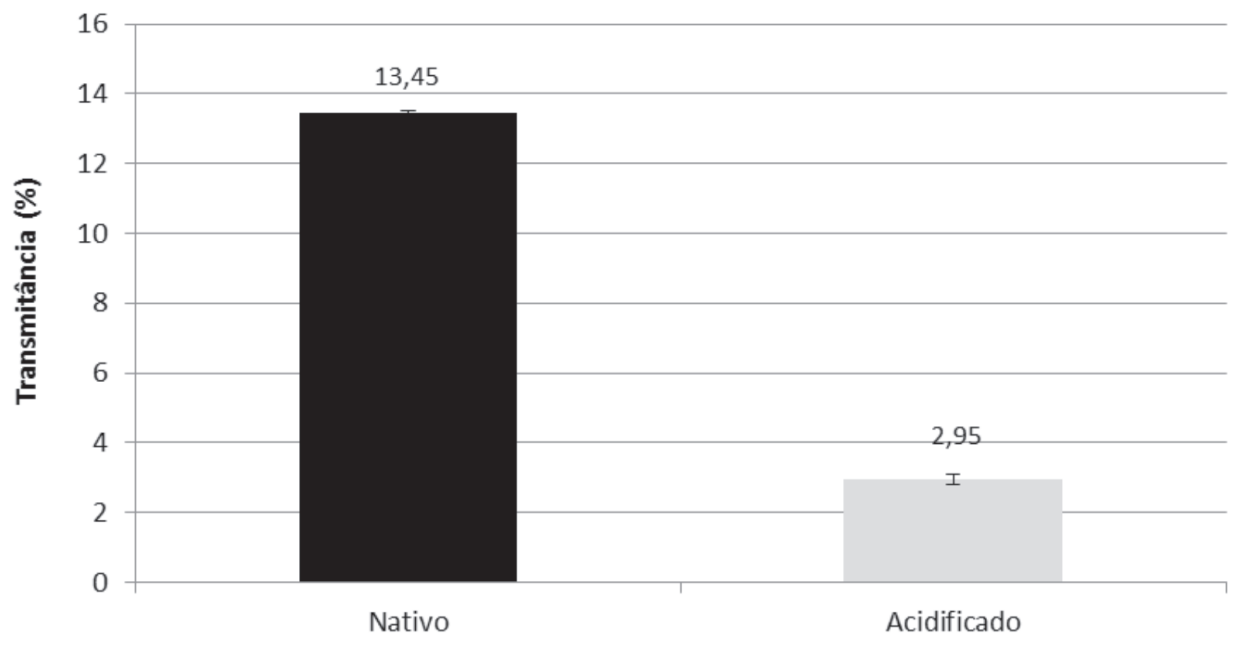

Figura 2: Transparência da pasta dos amidos nativo e acidificado das amêndoas dos caroços de manga (\% transmitância a 650 nm).

Tabela 3: Propriedades de pasta por RVA dos amidos nativo e acidificado do caroço de manga

\begin{tabular}{lcc}
\hline Parâmetros & Amido nativo & Amido acidificado \\
\hline Pico de viscosidade (cP) & $1.939,00 \pm 152,74^{\mathrm{A}}$ & $273,00 \pm 4,32^{\mathrm{B}}$ \\
Viscosidade mínima (cP) & $809,00 \pm 161,22^{\mathrm{A}}$ & $51 \pm 2,33^{\mathrm{B}}$ \\
Quebra de viscosidade (cP) & $1.130,00 \pm 8,48^{\mathrm{A}}$ & $222 \pm 10,11^{\mathrm{B}}$ \\
Viscosidade final $(\mathrm{cP})$ & $1.545,00 \pm 200,82^{\mathrm{A}}$ & $90,00 \pm 6,76^{\mathrm{B}}$ \\
Retrogradação $(\mathrm{cP})$ & $736,00 \pm 39,59^{\mathrm{A}}$ & $39,00 \pm 3,33^{\mathrm{B}}$ \\
Temperatura de pasta $\left({ }^{\circ} \mathrm{C}\right)$ & $76,72 \pm 0,03^{\mathrm{A}}$ & $76,32 \pm 0,75^{\mathrm{A}}$ \\
\hline
\end{tabular}

* Cada valor representa a média e o desvio-padrão de três determinações.

Diferença significativa entre colunas para uma mesma propriedade - letras maiúsculas diferentes. 
Isto ocorre porque durante o processo de acidificação, os íons hidroxônios $\left(\mathrm{H}_{3} \mathrm{O}^{+}\right)$ligam-se aos átomos de oxigênio das ligações glicosídicas, hidrolisando essas ligações. O ácido, então, gradualmente degrada as superfícies dos grânulos de amido, antes de entrar em seu interior. Ele quebra preferencialmente a região amorfa, uma vez que a região cristalina não é livremente acessível para ele, e isto faz que ela permaneça intacta. Como resultado, o percentual de cristalinidade relativa aumenta com a acidificação. Esse aumento da cristalinidade é o provável responsável pelo aumento da solubilidade e redução do intumescimento (Lawal $\&$ Adebowale, 2005).

Com relação à capacidade de absorção de água e óleo, o amido nativo apresentou maior valor para ambas. Kaur at al. (2004) obtiveram valores de CAA, de amidos nativos de caroços de manga, bem próximos aos resultados encontrados. Torruco-Uco \&Betancur-Ancona (2007) encontraram, em amido nativo do tubérculo makal (Xanthosoma yucatanensis), uma CAA de 19,2 g/100 $\mathrm{g}$, resultado inferior ao do amido do caroço de manga. Lawal \& Adebowale (2005) expuseram valores de CAA e CAO do amido nativo de "jack bean" bem menores do que os encontrados para o amido nativo desta pesquisa, não chegando nenhum dos dois valores a $3 \mathrm{~g} / 100 \mathrm{~g}$.

Singh et al. (2009) observaram que, assim como neste trabalho, o amido acidificado de "water chesnut" mostrou redução tanto da CAA quanto da CAO, em comparação com seu amido nativo. Muhammad et al. (2010) explicam que a hidrólise ácida basicamente reduz as capacidades de absorção de água e óleo, porque reduz as regiões amorfas dos grânulos do amido, diminuindo, assim, o número de sítios de ligação para água e óleo na molécula de amido.

Segundo Conto et al. (2011), a transparência da pasta pode variar de clara a opaca. Esta propriedade está relacionada com a dispersão da luz resultante da associação da amilose e de outros componentes dentro do amido. Após o cozimento da suspensão de amido, os grânulos intumescem e perdem a birrefringência, permitindo a passagem de luz, sendo possível, desta forma, detectar a sua transparência.

Nesta pesquisa, o amido nativo apresentou maior transmitância do que os demais amidos analisados. Isto pode ser explicado pelo fato de que os amidos modificados apresentavam alteração em sua coloração com relação a do amido nativo, variando de verde a amareloescura. Singh et al. (2007) verificaram, em diferentes variedades de arroz, que a transmitância da claridade da pasta variou entre 1,5 e $33,2 \%$. Estes autores relataram que as pastas de amidos podem ser menos translúcidas, por causa da presença de lipídeos complexados à amilose e resquícios de grânulos de amidos intumescidos. Abera
\& Rakshit (2003) relatam, ainda, que fatores como a presença de fibras, lipídeos e pigmentos pode diminuir a transparência das pastas de amido.

Os perfis viscoamilográficos dos amidos avaliados mostraram alterações, destacando-se que o amido acidificado apresentou menores valores para todos os parâmetros avaliados.

Conforme Leonel et al. (2005), a temperatura na qual os grânulos começam a inchar é denominada de temperatura de pasta e, para esta variável, os amidos nativos e acidificados não mostraram diferenças significativas. As viscosidades máximas (picos de viscosidade) dos amidos nativo e acidificado foram de $2047 \mathrm{cP}(176,46 \mathrm{RVU})$ a 273,00 (23,53 RVU).

Espinosa-Solis et al. (2009) encontraram, para o amido nativo da polpa de manga, variedade Tommy Atkins, e de banana "de vez" (em estado de maturação fisiológica), valores de pico de viscosidade maiores que os do amido nativo desta pesquisa, sendo estes, respectivamente, 194,1 RVU e 215,8 RVU. Rengsutthi \& Charoenrein (2011) verificaram para o amido nativo do caroço de jaca pico de viscosidade de 255,58 RVU. ElSaied et al. (1979) afirmam que a proteína pode atuar como barreira física para o intumescimento do amido, uma vez que os grânulos de amido são encaixados na matriz de proteína. Logo, o alto teor de proteína do amido nativo do caroço de manga $(5,60 \mathrm{~g} / 100 \mathrm{~g})$ pode justificar a baixa viscosidade de pico, comparada à de outras fontes amiláceas.

O amido nativo mostrou viscosidade mínima de 809 cP e quebra de viscosidade de $1130 \mathrm{cP}$. Espinosa-Solis et al. (2009) expuseram, tanto para o amido de polpa de maga como para o de banana, valores de quebra de viscosidade menores do que os encontrados para o amido nativo deste trabalho, sendo estes 50,2 RVU e 33,5 RVU, respectivamente. Rengsutthi \& Charoenrein (2011) verificaram para o amido nativo do caroço de jaca viscosidade mínima de 215,92 RVU e quebra de 39,67 RVU, sendo o primeiro valor maior e o segundo menor do que os observados nesta pesquisa. No entanto, Song et al. (2010), estudando propriedades viscoamilográficas de amido de milho, por RVA, obtiveram viscosidade mínima de $1201 \mathrm{cP}$ e quebra de viscosidade de $3246 \mathrm{cP}$, ou seja, apesar de a viscosidade mínima do amido analisado por estes autores ter sido um pouco superior à deste estudo, sua quebra de viscosidade foi bem superior à do amido nativo do caroço de manga, demonstrando maior fragilidade deste amido, quando cozido em água, que a do amido nativo desta pesquisa.

Espinosa-Solis et al. (2009) e Rengsutthi \& Charoenrein (2011) encontraram, ainda, valores de viscosidade final maiores que os do amido nativo deste estudo. Logo, um produto elaborado com o amido nativo 
desta pesquisa será um produto menos viscoso do que os elaborados com os demais.

Espinosa-Solis et al. (2009) encontraram, em seus amidos, valores de retrogradação de 95,2 RVU e de 141,7 RVU. Rengsutthi \& Charoenrein (2011) observaram, para o amido do caroço de jaca, retrogradação de 148,21 RVU. Estes trabalhos expõem valores maiores do que o obtido para o amido nativo desta pesquisa. Isto indica boa estabilidade do amido nativo de manga à retrogradação.

Shandu et al. (2007) relataram que, para diferentes espécies de amidos de milho ácido-modificados, ocorreram drásticas reduções dos valores de pico de viscosidade, viscosidade final, quebra de viscosidade e retrogradação, quando comparado com os do amido nativo. De acordo com os mesmo autores, a hidrólise ácida gera esta redução drástica do pico de viscosidade, por causa da redução das cadeias que compõem o amido.

Singh et al. (2007) verificaram, no amido acidificado de "water chestnut" que a medida que se aumentava a concentração da solução ácida, seus amidos acidificados apresentavam redução do pico de viscosidade, da viscosidade mínima, da viscosidade final, da retrogradação e da temperatura de pasta. Isto corrobora os resultados encontrados para o amido acidificado deste estudo. Os menores valores de quebra de viscosidade e retrogradação, para o amido acidificado do caroço de manga, sugerem tanto maior estabilidade à agitação mecânica quanto menor tendência à retrogradação, quando comparado com os do amido nativo.

\section{CONCLUSÕES}

O extrato amiláceo nativo apresentou percentual significativo de amido; no entanto, também expôs quantidades relevantes de lipídeos e proteínas, em sua composição, que foram componentes decisivos nas propriedades funcionais estudadas nesta pesquisa.

$\mathrm{O}$ amido nativo revelou baixa resistência à elevação de temperatura, mostrando intumescimento máximo a $75^{\circ} \mathrm{C}$, com relevante solubilização a esta temperatura. Este amido caracterizou-se, ainda, por consideráveis valores de capacidade de absorção de água e óleo, maior transparência de pasta, baixo pico de viscosidade quando comparado com outras fontes amiláceas, interessante resistência à agitação mecânica (quebra) e baixa tendência a retrogradar. Pode ser indicado para compor sopas desidratadas, já que apresenta baixa temperatura de formação de gel, e em produtos cárneos, por apresentar boa capacidade de reter água.

O amido acidificado (hidrolisado) mostrou o menor intumescimento e a maior solubilidade, com relação à temperatura, e os menores valores de capacidade de absorção de água e óleo. Foi o amido que apresentou o menor pico de viscosidade, resistência à agitação mecânica (quebra) e tendência a retrogradar. Esse amido pode ser utilizado em alimentos congelados e refrigerados, por essa baixa tendência em retrogradar, na indústria de balas, por sua baixa viscosidade e retrogradação, e em sopas desidratadas (baixa temperatura de formação do gel).

\section{REFERÊNCIAS}

Abera S \& Rakshit KS (2003) Comparison of physicochemical and functional properties of cassava starches. Starch - Stärke, 55:287296.

Adebowale kO, Olu-owolabi BI, Olawumi EK \& Lawal OS (2005) Functional properties of native, physically and chemically modiûed breadfruit (Artocarpus artilis) starch. Industrial Crops and Products, 21:343-351.

Anggraini V, Sudarmonowat E, Hartati NS, Suurs L \& Visser RGF (2009) Characterization of Cassava Starch Attributes of Different Genotypes. Starch-Stärke, 6:472-481.

Batista WP, Silva CEM \& Liberato MC (2010) Propriedades químicas e de pasta dos amidos de trigo e milho fosforilados. Ciência e Tecnologia de Alimentos, 30:88-92.

Beemiller J \& Wistler R (2009) Starch: Chemistry and Tecnology. $3^{\text {a }}$ ed. West Lafayette, Academic Press. 894p.

Bello-Pérez LA, Montealvo MGM \& Acevedo EA (2006) Almidón: definición, estructura y propriedades. In: Lajolo FM \& Menezes EW (eds.) Carboidratos em alimentos regionais iberoamericanos. São Paulo, Edusp. 646p.

Bertolini AC (2010) Starchs: Characterizarion, Properties and Applications. Washington, CRC Press. 288p.

Beuchat LR (1977) Functional and electrophoretic characteristics of succinylated peanut flour protein. Journal of Agriculture and Food Chemistry, 25:258-261.

Brandão MCC, Maia GA, Lima DP, Parente EJS, Campello CC \& Nassu RT (2003) Análise físico química, microbiológica e sensorial de frutos de manga submetidos à desidratação osmótico solar. Revista Brasileira de Fruticultura, 25:34-39.

Conto LC, Plata-Oviedo MSV, Steel CJ \& Chang YK (2011) Physicochemical, morphological, and pasting properties of Pine nut (Araucaria angustifolia) starch oxidized with different levels of sodium hypochlorite. Starch - Stärke, 63:198-208.

Costa JG \& Santos CAF (2004) Cultivares. In: Cultivo da Mangueira. Sistema de Produção II: Embrapa Semi-Árido. Disponível em: <http:/ /sistemasdeproducao.cnptia.embrapa.br/FontesHTML/Manga/ CultivodaMangueira/cultivares.htm>. Acessado em: 29 de novembro de 2012.

Craig SAS, Maningat CC, Seib PA \& Hoseney RC (1989) Starch Paste Clarity. Cereal Chemistry, 66:173-182.

El-Saied HM, Ahmed EA, Roushdi M \& El-Attar W (1979) Gelatinization, pasting characteristics and cooking behaviour of Egyptian rice varieties in relation to amylose and protein contents. Starch - Stärke, 31:270-274.

Espinosa-Solis V, Jane J \& Bello-Perez AL (2009) Physicochemical Characteristics of Starches from Unripe Fruits of Mango and Banana. Starch - Stärke, 61:291-299.

Hashim DB, Moorthy SN, Mitchell JR, Hill SE, Linfoot KJ \& Blanshard JMV (1992) The effect of low levels of antioxidants on the sweeling and solubility of cassava starch. Starch - Stärke, 44:471-475. 
Instituto Adolfo Lutz (2008) Métodos Físico-Químicos para análise de Alimentos: normas analíticas do Instituto Adolfo Lutz. São Paulo. Disponível em: <http://www.ial.sp.gov.br/ index.php?option=com_remository\&Itemid=20>. Acessado em: 25 de janeiro de 2012.

Kaur M, Singh N, Sandhu KS \& Guraya HS (2004) Physicochemical, morphological, thermal, and rheological properties of starches separated from kernels of some Indian mango cultivars (Mangifera indica L.). Food Chemistry, 85:131-140.

Lawal OS \& Adebowale KO (2005) Physicochemical characteristics and thermal properties of chemically modified jack bean (Canavalia ensiformes) starch. Carbohydrates Polymers, 60:331-341.

Leach HW, McCowen LD \& Schoch TJ (1959) Structure of the starch granule. I. Swelling and solubility patterns of various starches. Cereal Chemistry, 36:534-544.

Leonel M, Ferrari TB, Sarmento SBS \& Oliveira MA (2005) Planting time, developmental stages and characteristics of roots and starch of Phachyrhizus ahipa. Ciência e Tecnologia dos Alimentos, 62:528533.

Loos PJ, Hood LF \& Graham HD (1981) Isolation and Characterization of Starch from Breadfruit. Cereal Chemistry, 54:282-286.

Muhammad K, Hussin F, Man YC, Ghazali HM \& Kennedy JF (2010) Effect of $\mathrm{pH}$ on phosphorylation of sago starch. Carbohydrates Polymers, 42:85-90

Osundahunsi OF \& Mueller R (2011) Functional and dynamic rheological properties of acetylated starches from two cultivars of cassava. Starch - Stärke, 63:03-10.

Rengsutthi K \& Charoenrein S (2011) Physicochemical properties of jackfruit seed starch (Artocarpus heterophyllus) and its application as a thickener and stabilizer in chilli sauce. Food Science and Technology, 44:1309-1313.

Serrano PO \& Franco CML (2005) Modificações hidrotérmicas ("Annealing") e hidrólise enzimática do amido de mandioca. Brazilian Journal of Food Technology, 8:220-232.
Shandu KS, Singh N \& Lim TS (2007) Functional properties of normal, waxy and sugary corn starch. Journal of Food Science and Technology, 44:565-571.

Singh GD, Bawa AS, Riar CS \& Saxena DC (2009) Influence of heatmoisture treatment and acid modifications on physicochemical, rheological, thermal and morphological characteristics of indian water chestnut (Trapa natans) starch and its application in biodegradable films. Starch - Stärke, 61:503-513.

Singh H, Chang YH, Sodhi NS \& Singh N (2011) Inûuence of prior acid treatment on physicochemical and structural properties of acetylated sorghum starch. Starch - Stärke, 63:291-301.

Singh N, Nakaura Y, Inouchi N \& Nishinari K (2007) Fine Structure, Thermal and Viscoelastic Properties of Starches Separated from Indica Rice Cultivars. Starch - Stärke, 9:135-147.

Song X, Zhu W, Li Z \& Zhu J (2010) Characteristics and application of octenyl succinic anhydride modiûed waxy corn starch in sausage. Starch - Stärke, 62:629-636.

Tongdang T (2008) Some properties of starch extracted from three Thai aromatic fruit seeds. Starch/Stärke, 60:199-207.

Torruco-Uco J \& Betancur-Ancona D (2007) Physicochemical and functional properties of makal (Xanthosoma yucatanensis) starch. Food Chemistry, 101:319-326.

Wang YJ \& Wang L (2003) Physicochemical properties of common and waxy corn starches oxidized by different levels of sodium hypochlorite. Carbohydrates Polymers, 52:207-217.

Wang YJ, Truong V \& Wang L (2003) Structures and rheological properties of corn starch as affected by acid hydrolysis. Carbohydrates Polymers, 52:321-333.

Zambrano F \& Camargo CRO (2001) Otimização das condições de hidrólise ácida de amido de mandioca para obtenção de substituto de gordura. Brazilian Journal of Food Technology, 4:147-154.

Zein RE, El-Bagoury AA \& Kassab HE (2005) Chemical and nutritional studies on mango seed kernels. Journal of Agricultural Science, 30:3285-3289. 